\title{
A Fast Method for Obtaining a "Continuous" Sample of the Inversion-Recovery Curve Using Spatially Swept Adiabatic Inversion
}

\author{
JAMES G. PIPE \\ Department of Radiology, University of Michigan Medical Center, \\ 1500 East Medical Center Drive, Ann Arbor, Michigan 48109
}

Received October 7, 1991; revised February 25, 1992

The measure of the longitudinal, or $T_{1}$, relaxation time in nuclear magnetic resonance experiments is a common diagnostic tool. There have been several methods proposed for measuring this parameter. Most of these methods measure the recovery of longitudinal magnetization after inversion or saturation. Conventional $\left(180^{\circ}-T_{i}-\right.$ $90^{\circ}$ ) experiments measure the signal strength for a single temporal point $T_{i}$ on the recovery curve. These protocols are typically long, due to the necessarily long times between NMR experiments (TR), which must be on the order of five times the $T_{1}$ constant or more. Many new techniques require only one experiment to obtain several points on the relaxation curve $(1-3)$. These are based on a technique proposed by Look and Locker (4) and later by Kaptein et al. (5) in which each data-collection experiment involves one inversion followed by $N$ small-tip-angle RF pulses which "probe" the signal strength over time. The change in signal strength for each of the $N$ acquisitions is correlated with the difference in $T_{i}$, allowing the relaxation rate to be sampled at $N$ points in one data-collection period. Another group of $T_{1}$ methods is based on the equilibration of magnetization under rapid excitation $(6,7)$. These methods are also much faster than conventional inversion-recovery methods. A common quality of all of the above techniques is that they give a limited number of discrete measures of longitudinal relaxation.

The method described in this Note is able to give a much greater sampling of the relaxation curve in a single experiment. It correlates $T_{i}$ with a spatial dimension by adiabatically inverting spins in the presence of a magnetic-field gradient. An example of the pulse sequences used is shown in Fig. 1. The change in frequency of the inverting RF is constant (and adiabatic) over time, thus causing an inversion of spins which is temporally linear with distance in the spatial dimension. If the region of the sample which is inverted by this pulse is homogeneous in $T_{1}$, then the inversion-recovery curve over time will be represented as a continuous inversion-recovery curve over space. The amount of the relaxation curve visible in the experiment is equal to the sweep time. A simple spin-echo (or gradient-echo) experiment with a readout gradient in the same direction as the inversion will allow one to discriminate points along the inversion-recovery curve at high resolution.

The choice of adiabatic inversion parameters is governed by three major equations. These equations are given with respect to the effective field shown in Fig. 2, where $H_{1}$ 


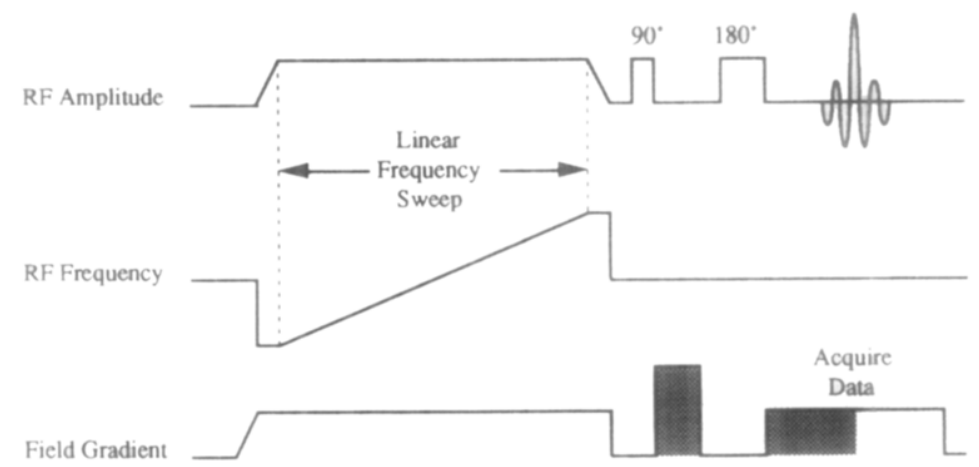

FIG. 1. Pulse sequence diagram for the proposed technique. Gray areas show balanced gradients for refocusing of echoes.

is the amplitude of the RF pulse, $\Delta \omega$ is the instantaneous offset frequency of a spin from the RF resonance, $\gamma$ is the gyromagnetic ratio for the spin, $G$ is the constant amplitude of the field gradient, and $x$ is the spatial distance. The first condition is necessary for adiabaticity $(8)$, and is given by

$$
H_{1}^{2} \gg\left|\frac{(d / d t)(G x)}{\gamma}\right|=\left|\frac{G}{\gamma} \frac{d x}{d t}\right| .
$$

Here, $d x / d t$ is the rate at which the RF "sweeps" over distance and is equal to the inversion width divided by the sweep time. Theoretically this condition can easily be met, since long sweep times (large $d t$ ) are desired to sample an appropriate amount of the relaxation curve. Practically, the digital resolution necessary to define a parabolic phase (i.e., linear frequency) function over long times was a confining limit in our experiments. The second condition determines the "edge effects" of the adiabatic sweep; for an effective full-passage adiabatic inversion, the RF must sweep from far above resonance to far below resonance for every spin starting with its magnetization in equilibrium along the longitudinal axis. "Far" above and below are defined relative

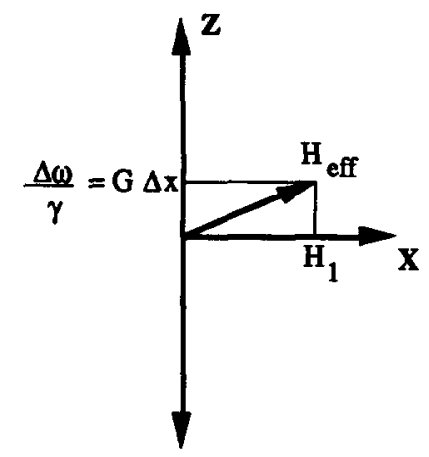

FIG. 2. Effective field, showing $H_{\mathrm{eff}}$ in the coordinate system rotating at $\omega_{\mathrm{RF}}$. The presence of a magnetic field gradient allows off-resonance frequency to be directly translated to distance. 


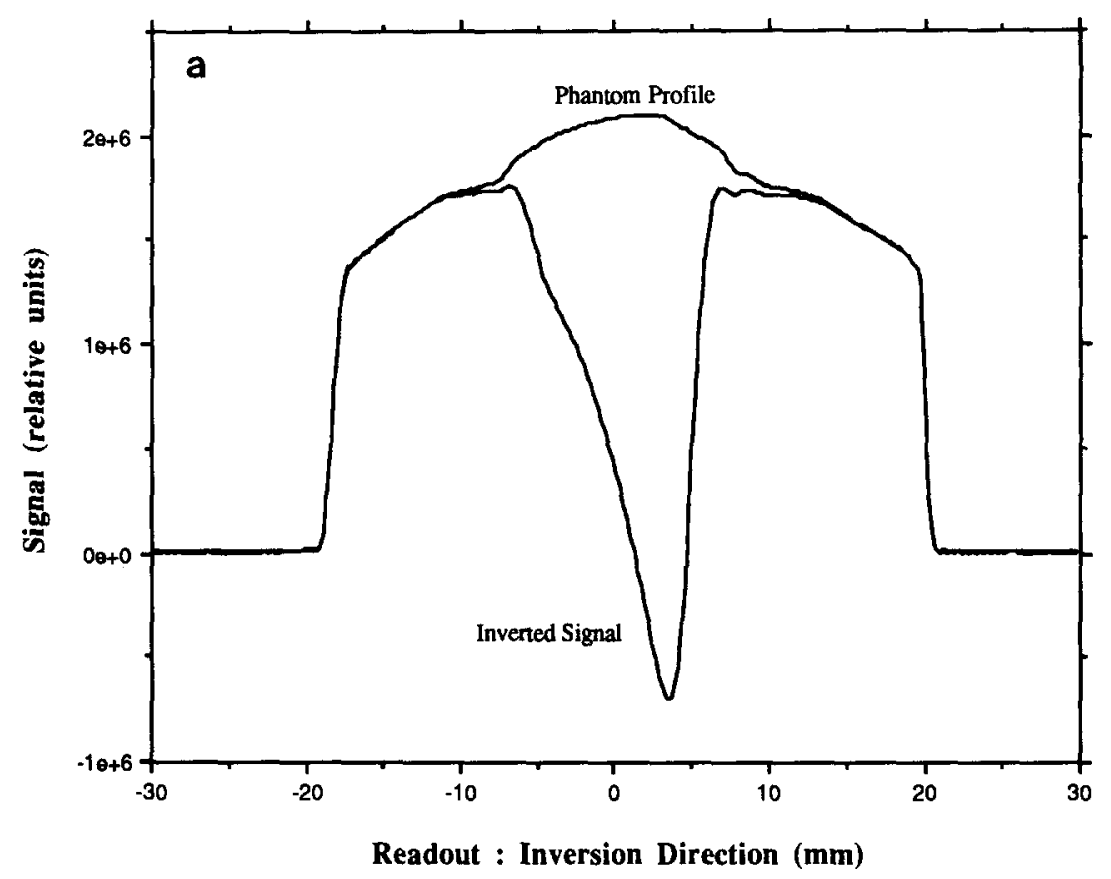

FIG. 3. Single-exponential relaxation phantom results. In (a), the adiabatic sweep was across $10 \mathrm{~mm}$ in $50 \mathrm{~ms}$, and the phantom had an independently measured $T_{1}$ of $28 \mathrm{~ms}$. In (b), the sweep time was $100 \mathrm{~ms}$ and the $T_{1}$ was $122 \mathrm{~ms}$. For both phantoms, the adiabatic sweep started at the left and ended at the right edge of the inversion profile. The two inverted data sets were normalized with the noninverted data sets, and the appropriate section of the relaxation curves was extracted; they are plotted against calculated inversion times in (c) along with the predicted relaxation curves.

to the strength of the RF pulse. This condition is met for all spins farther than $\Delta x$ away from the edges of the spatial inversion, where

$$
H_{1} \ll|G \Delta x| \text {. }
$$

All points within $\gamma G \Delta x$ of the start and end of the frequency sweep will have experienced unique RF and should not be included in the recovery profile. It is particularly significant to minimize this effect in the last part of the sweep, where the inversion time is smallest and the largest dynamic ranges in the signal are present. Note in Fig. 1 that just before and after the frequency sweep, the amplitude of the RF is ramped, so that cleaner edges are obtained and this condition is somewhat relaxed for those spins near the border. The last condition is necessary if relaxation effects are to be considered negligible $\left(8\right.$, p. 66). Obviously, $T_{1}$ effects are desired, but to avoid $T_{2}$ effects, the equation

$$
H_{1} \ll\left|T_{2} \frac{d}{d t}(G x)\right|=\left|T_{2} G \frac{d x}{d t}\right|
$$

must hold. This last condition is desirable but not necessary. If it does not hold, then the recovery data will have attenuated amplitude and will not extrapolate to $-M_{0}$ at $T_{i}=0$ or $M_{0}$ at $T_{i}=\infty$. 

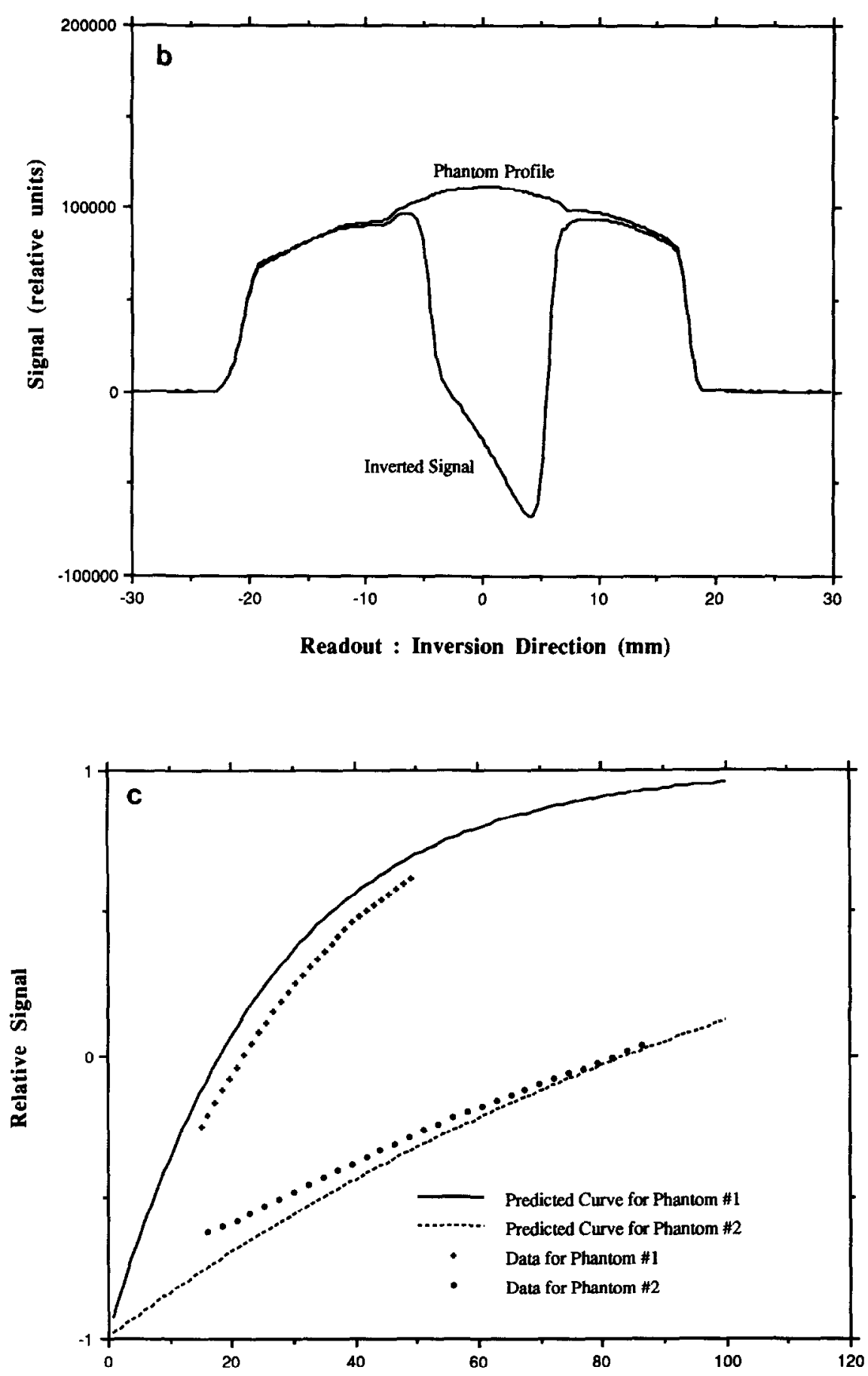

Time after Inversion (ms)

Fig. 3-Continued 


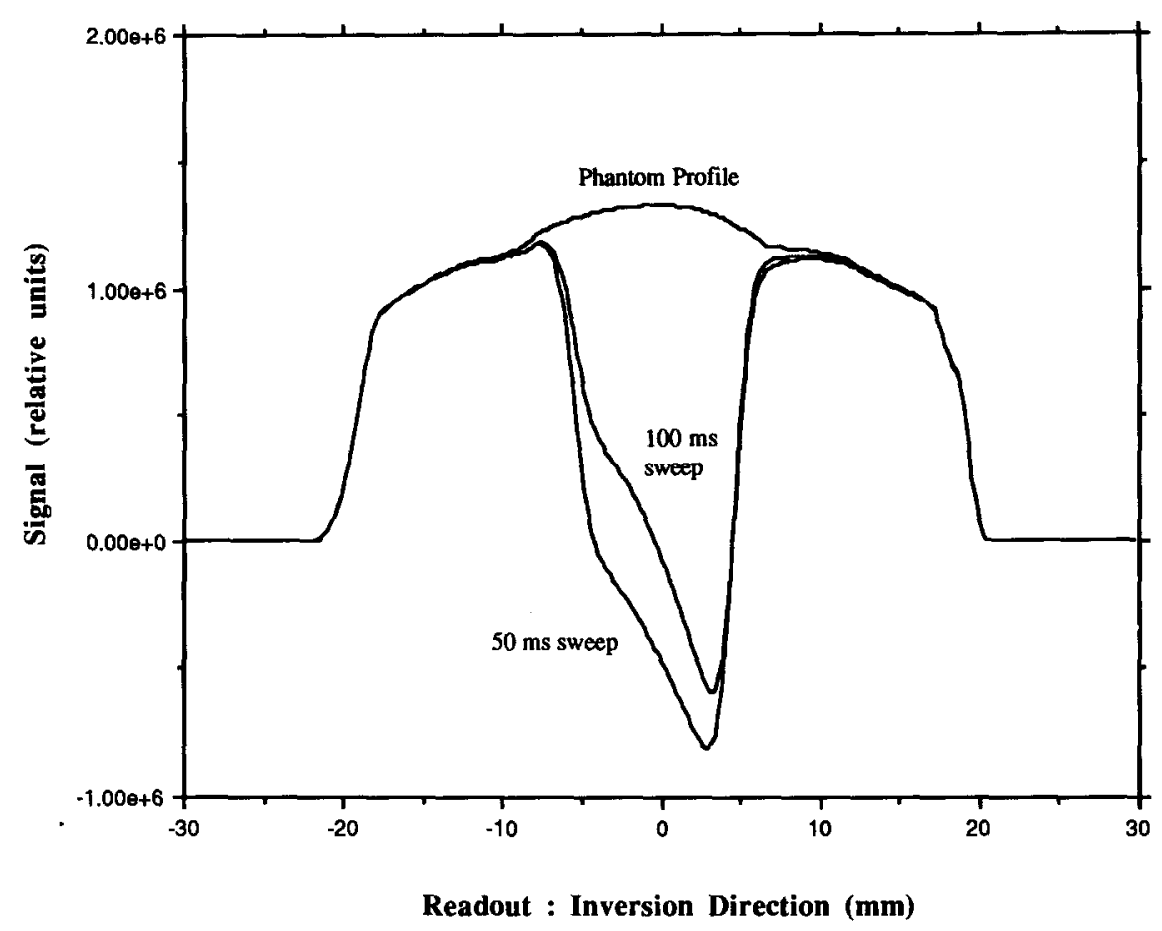

FIG. 4. Biexponential relaxation phantom results. The noninverted profile of the dual- $T_{1}$ phantom is shown along with inverted profiles with a sweep time of 50 and $100 \mathrm{~ms}$.

The major advantage of this technique is that a very fine sampling of the recovery curve can be acquired with a minimal number of experiments. Typically a noninverted spectrum (with the inversion pulse turned off) must be collected to account for inhomogeneity of the RF coil, and phase cycling of the adiabatic pulse is desired to remove residual transverse magnetization at the two ends of the frequency sweep. Thus at least three experiments are needed to obtain a relaxation curve, although signal-to-noise ratio requirements may require averaging a larger number of experiments. Because the inversion is adiabatic, it is independent of the RF homogeneity over a large range of $H_{1}$, and most points in the sample will be cleanly inverted. As stated above, the inversion will not be complete at the beginning and ending frequencies of the adiabatic sweep, and the NMR signal at these points will deviate from the desired representation of the temporal relaxation curve.

Two experiments were done to verify the ability of this technique to measure $T_{1}$. All experiments were performed on a 2 T CSI Omega NMR system (General Electric, Fremont, California). The first experiment involved the measure of $T_{1}$ relaxation curves of two phantoms using nonselective $90^{\circ}$ and refocusing $180^{\circ}$ pulses preceded by the adiabatic inversion. The phantoms consisted of distilled water doped with (different amounts of) $\mathrm{CuSO}_{4}$. Conventional $\left(180^{\circ}-\tau-90^{\circ}\right)$ inversion-recovery experiments were used to measure eight points ( 32 averages each) along the $T_{1}$ relaxation curve for both phantoms individually, and a single exponential was fitted to each data 


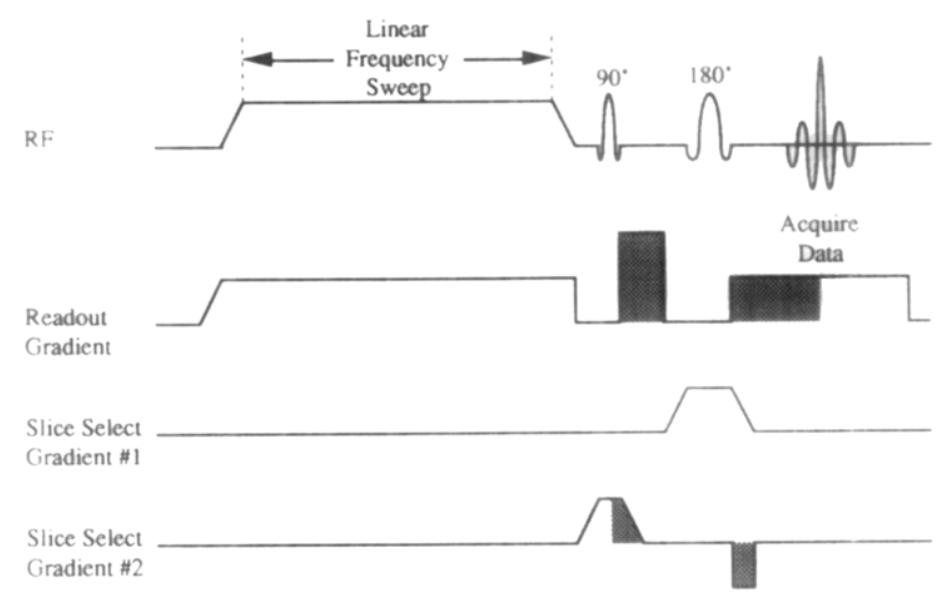

FIG. 5. Column imaging version of pulse sequence shown in Fig. 1. Gray areas show balanced gradients for refocusing of echoes.

set using a least-mean-squares (lms) fitting routine (9) included in the Omega software (General Electric). The calculated $T_{1}$ values were 28 and $122 \mathrm{~ms}$ for phantoms " 1 " and " 2 ," respectively. The $T_{1}$ curve was then measured by the proposed method for each of the phantoms ( 32 averages per curve); these data were normalized with a noninverted profile of the phantom ( 32 averages) and fitted to a single exponential. Calculated $T_{1}$ values were 29 and $135 \mathrm{~ms}$ for phantoms 1 and 2, respectively. The spatial profiles of each phantom with and without inversion are shown in Fig. 3; also shown are the chosen data along with the predicted relaxation curves. All of the adiabatic RF pulses in this experiment were at $0.2 \mathrm{G}$, and the field gradient during inversion was $1 \mathrm{G} / \mathrm{cm}$. The system parameters of Eqs. [1]-[3] were compromised because of the inability to slowly sweep over large frequency ranges due to the waveform resolution limits of the system. A dual- $T_{1}$ experiment was performed using both phantoms together, but the fit was very unreliable. It is believed that this failure was partly due to the compromise in Eqs. [1] and [2] because of system limitations. The spatial profiles of this experiment are shown in Fig. 4.

The second experiment demonstrated the use of this method in a column imaging mode (as shown in Fig. 5) for in vivo determination of the $T_{1}$ of a spatially localized volume of liver in a rat. A standard imaging protocol was used to decide on a homogeneous region (seen in Fig. 6) appropriate for the technique; the proposed method was then performed to obtain a $T_{1}$ relaxation curve for the volume. Conventional inversion recovery determined the local $T_{1}$ of the liver to be $610 \mathrm{~ms}$, compared with $587 \mathrm{~ms}$ as given by the proposed method.

A discrete version of this technique was presented by McVeigh et al. (10) and was implemented by successively inverting thin planes of spins orthogonal to the readout direction, making the inversion time at readout different for cach intersection of an inverted plane and the readout direction. This method has the advantage of arbitrary inversion times for each plane, but gives a much more limited sampling of the relaxation curve than the proposed technique. The proposed technique is analogous to the method 

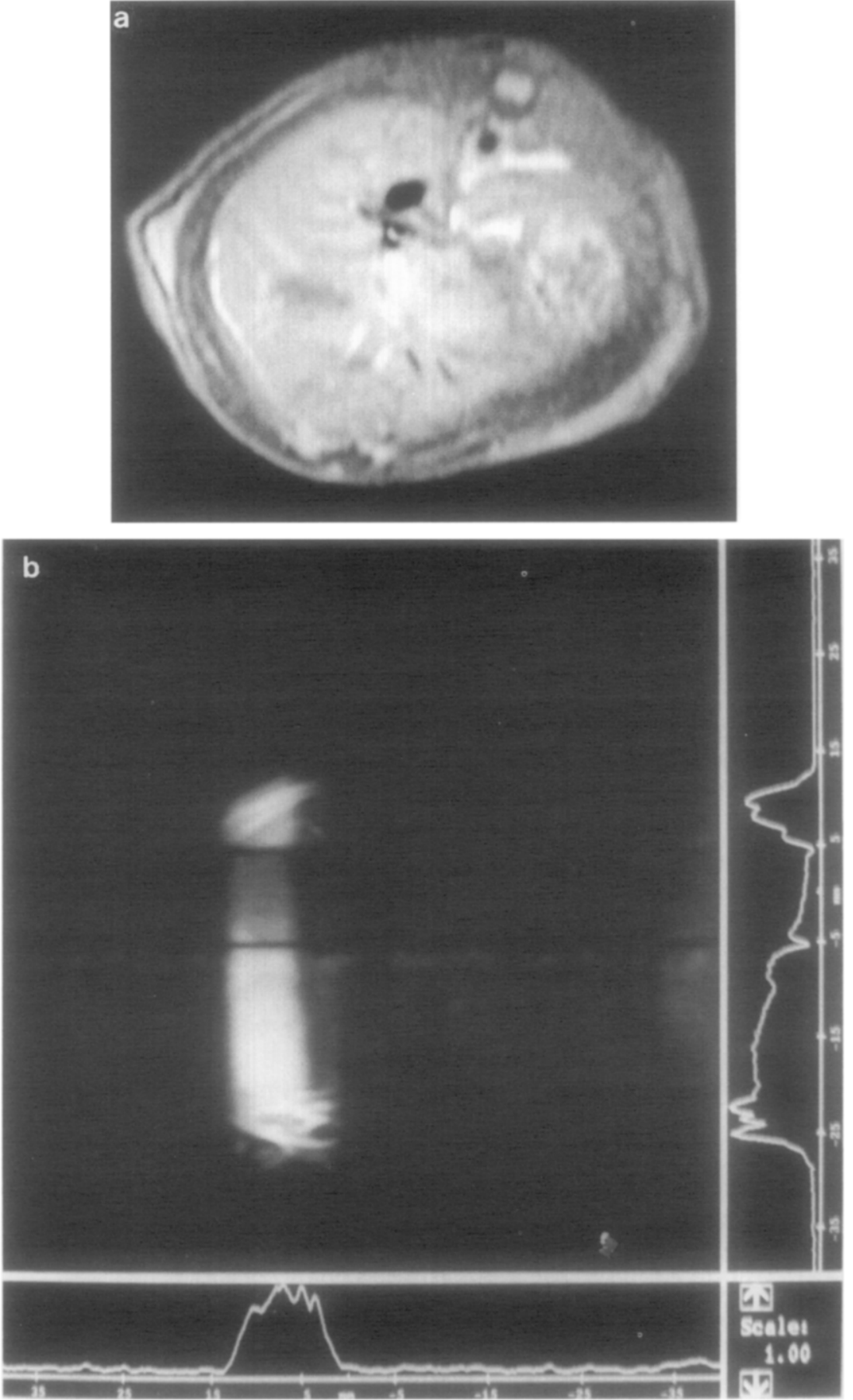

FIG. 6. (a) Axial image of rat liver used for the $T_{1}$ experiment. (b) The column from (a) used for the experiment. The dark band in (b) is due to the adiabatic inversion; a profile of this inversion can be seen along the side of the image. 
of Swanson (11), in which a gradient is used to obtain a finely sampled measure of off-resonance saturation due to cross-relaxation in aqueous heterogeneous materials. The effect of relaxation during adiabatic inversions has been noted elsewhere $(12,13)$ but was treated as an unwanted artifact. This technique exploits that "artifact" by using parameters such as long sweep widths which cause more pronounced relaxation effects during the inversion. The proposed method should be useful when fast measures of $T_{1}$ are necessary, or when multiple $T_{1}$ values exist within a single homogeneous sample, since many points along the recovery curve must be measured to provide unique multiexponential solutions to the data $(14,15)$. The experimental conditions in this Note did not fully satisfy Eqs. [1]-[3], and therefore it is expected that this method will produce more satisfactory results given the ability to adiabatically sweep over large frequency ranges for long periods of time.

\section{ACKNOWLEDGMENT}

The author thanks Scott Swanson for helpful discussions concerning the implementation of this method.

\section{REFERENCES}

1. I. R. Young, A. S. Hall, ANd G. M. Bydder, Magn. Reson. Med. 5, 99 (1987).

2. A. P. Crawley and R. M. Henkelman, Magn. Reson. Med. 7, 23 (1988).

3. G. BRiX, L. R. SCHAD, M. DeImling, AND W. J. Lorenz, Magn. Reson. Imaging 8, 351 ( 1990 ).

4. D. C. LOOK AND D. R. LOCKER, Rev. Sci. Instrum. 41, 250 (1970).

5. R. Kaptein, K. DiJKstra, AND C. E. TARr, J. Magn. Reson. 24, 295 (1976).

6. K. A. Christensen, D. M. Grant, E. M. Schulman, and C. Walling, J. Phys. Chem. 78, 1971 (1974).

7. J. Homer AND M. S. BeEvers, J. Magn. Reson. 64, 287 (1985).

8. A. Abragam, "Principles of Nuclear Magnetism," p. 65, Oxford Univ. Press; New York, 1961.

9. J. C. MacDonald, J. Magn. Reson. 38, 381 (1980).

10. E. MCVeigh, A. Yang, And E. Zerhouni, Med. Phys. 17, 131 (1990).

11. S. D. SWanson, J. Magn. Reson. 95, 615 (1991).

12. P. A. Gowland, M. O. Leach, AND J. C. ShaRP, Magn. Reson. Med. 12, 261 (1989).

13. J. M. Bohiten, R. BurghaRdt, M. ReY, AND G. BodenHausen, J. Magn. Reson. 90, 183 (1990).

14. R. J. S. Brown, J. Magn. Reson. 82, 539 (1989).

15. K. P. WhitTall, J. Magn. Reson. 84, 134 (1989). 